\title{
Téoros
}

Revue de recherche en tourisme

\section{Petits transports et grand tourisme}

\section{Pierre Bélec}

Volume 6, numéro 1, mars 1987

Tourisme et transports

URI : https://id.erudit.org/iderudit/1080526ar

DOI : https://doi.org/10.7202/1080526ar

Aller au sommaire du numéro

Éditeur(s)

Université du Québec à Montréal

ISSN

0712-8657 (imprimé)

1923-2705 (numérique)

Découvrir la revue

Citer cet article

Bélec, P. (1987). Petits transports et grand tourisme. Téoros, 6(1), 35-36. https://doi.org/10.7202/1080526ar d'utilisation que vous pouvez consulter en ligne.

https://apropos.erudit.org/fr/usagers/politique-dutilisation/ 


\section{Petits transports et grand tourisme}

Perçu comme marginal il y a quelques années, le tourisme des grandes randonnées en plein air poursuit son développement de manière soutenue.

par Pierre Bélec* *

\begin{abstract}
"Il y a dix ans, on les connaissait tous par leur nom ceux qui faisaient des longues randonnés de deux jours et plus à vélo. Aujourd'hui, ce n'est certainement plus le cas". C'est Louise Roy du Tour de l'Ile et de Vélo-Québec qui résume ainsi toute la distance qui nous sépare de ce qui se passait il y a seulement cinq ou dix ans, dans le petit monde des grands excursionnistes du plein air.
\end{abstract}

Mème son de cloche à la Fédération québécoise du canot-camping, à la Fédération québécoise de la montagne et de la Fédération du camping-caravaning. Phénomène récent, difficile à quantifier, mais unaniment observế par les parrains du plein air québécois: nos bords de route, nos rivières, nos sentiers sont très visiblement fréquentés par des gens qui parcourent d'assez longues distances.

Des familles en canot...

Et qui trouve-t-on dans les canots qui se ballottent chaque été sur les $30,000 \mathrm{~km}$ de rivière aux difficultés recensées et dont $20,000 \mathrm{~km}$ sont déjà cartographiés? Près de $30 \%$ de familles, qui partent régulièrement pour des excursions de fin de semaine, ou de trois jours. Donc, un homme, une femme avec un ou deux enfants.

Et ils sont nombreux. A cux seuls les pares et réserves comptent 100,00 utilisateurs, soit, d'après la fréquence annuelle de pratique des usagers des parcs, environ 75,000 personnes. Mais ce ne serait encore que la pointe de l'iceberg... car les rivières les plus intéressantes sont situées en dehors des parcs et réserves. En fait, Pierre Trudel, directeur général de la fédération, estime à 300,000 le nombre de canot-campeurs québécois, sur le total des 700,000 utilisateurs de la "flotte" québécoise, évaluée à 200,000 canots.

Bien sûr, les "granola boys" des années 70 vivent encore sous les traits d'une autre génération. Adeptes des expéditions de 10 jours, moins épris de retour à la nature, ils aiment se lancer sur des rivières difficiles, dans des canots bourrés de matériaux de flottaison ajoutée... pour le moment. Car

*Observateur du phênomêne plein air depuis une quinzaine d'annóes, I'auteur est conselllar en communication publique chez BCP/Synergie.

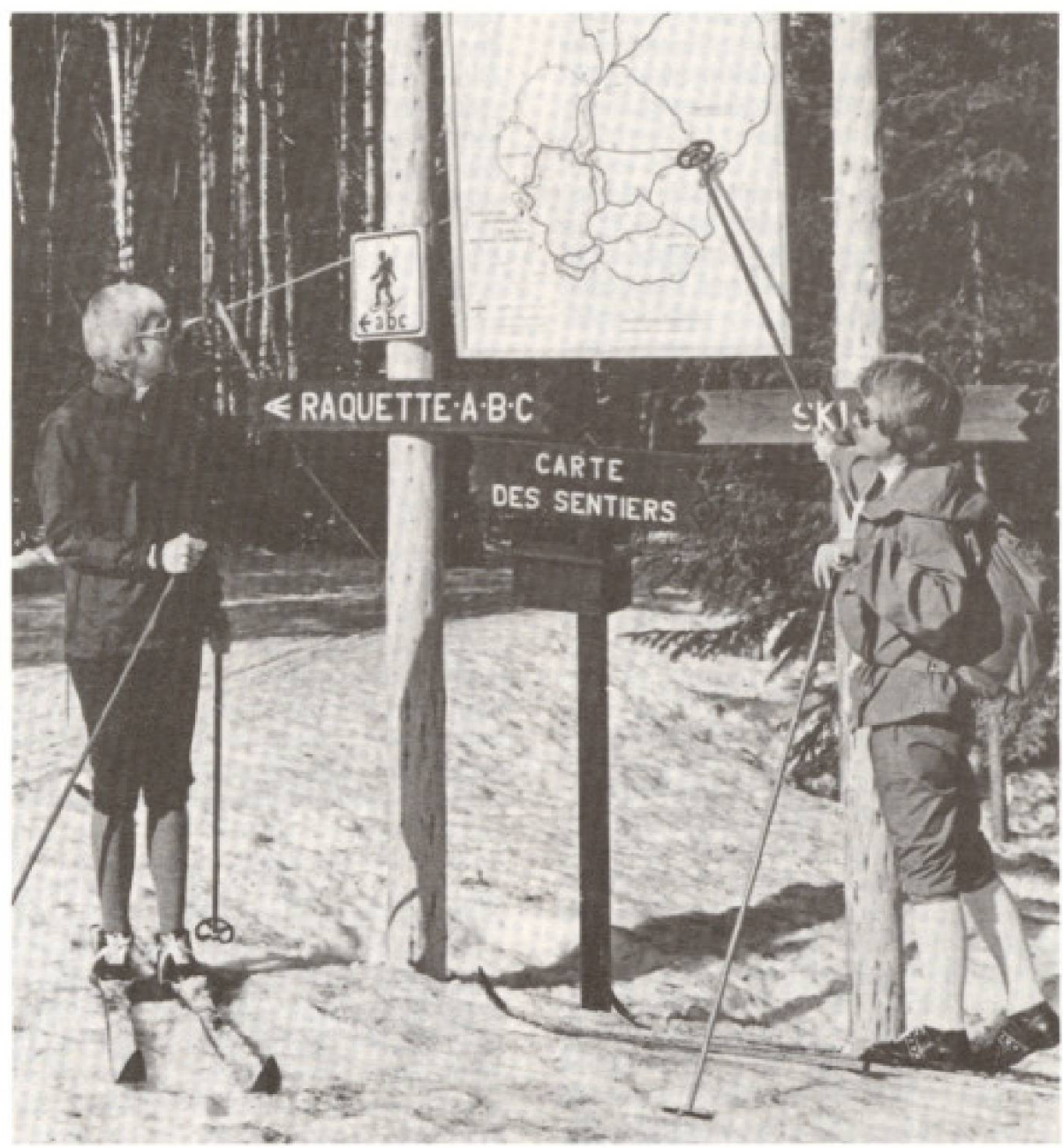

ils se rangeront sans doute à leur tour sur les traces de la génération précédente pour passer lentement, maman en proue, junior au milieu, vers des eaux moins tourmentées. Et se joindre aux milliers d"autres qui vivent cette importante sous-culture touristique du canotage sur les rivières du Québec.

\section{... et des baby boomers en tente roulotte}

C'est peut-être en camping et caravaning que la génération des "baby boomers" se manifeste le plus bruyamment. Acheteurs de petites remorques ou de véhicules motorisés, les 40 ans écument actuellement les salles de montre, épuisent les stocks... au point que les vendeurs de véhicules récréatifs seraient prêts à occuper tout le plancher des prochains Salons du Camping. On assiste présentement, d'après Irman Bolduc, directeur général de la Fédération de camping et de caravaning à une stagnation du camping d'occupation saisonniere et à un développement fulgurant du camping itinérant. En achetant un véhicule récréatif, les gens achètent leur autonomie récréotouristique et la promesse de nombreuses découvertes: en nature comme en collectivitế de vacances. Les campeurs se sont remis à la découverte du Québec; ils se maintiennent à l'intérieur d'une vaste zone touristique qui englobe les Maritimes et l'Ontario. Quant aux groupes organisés, il 
$n$ 'est pas rare de les voir voyager en convois de 150 unités et plus.

Soixante pour cent des lecteurs du Magazine Vélo-Québec, ils. sont plus de 7,000, ont fait des excursions à bicyclette de deux jours et plus. Mais ce n'est sans doute, ici encore, que la pointe du iceberg, les gens regroupés par une fédération ne représentant qu'une portion des pratiquants. Car le parc de vélos du Québec s'établit à environ 3,000,000 unites. Et l'on ne voit pas encore le bout de la révolution sur deux roues, puique les chiffres de vente augmentent sans cesse: on vend $30 \%$ plus de bicyclettes ces années-ci qu'il y a cinq ans. Les pistes cyclables des villes invitent à aller voir ailleurs, à tenter l'aventure; tout comme le Tour de l'Ile, ou $50 \%$ des 15,000 inscrits de $1986 \mathrm{n}^{\dagger}$ avaient encore jamais fait $60 \mathrm{~km}$ dans une journée.

De fait la carte du Québec cyclable se vend bien depuis déjà quelques années et le réseau des petites auberges à prix modique et de l'hébergement à la ferme signalent à Vélo-Québec que de plus en plus de leurs hôtes arrivent... et repartent à bicyclette. Mẻme phénomène dans les terrains de camping.

Sur deux roues comme en canot ou a ski, le sexe fort n'est plus tout à fait roi et maitre, puisque les femmes constituent actuellement au moins $35 \%$ de la clientèle. Et cette proportion $s^{4}$ inverse parfois en faveur des femmes dans les voyages de plein air organises.

\section{Des amateurs de neige}

Au royaume de l'hiver, il y a aussi eu des changements, L'an dernier, 85 groupes ont fait à ski les sept jours de la grande traversée de Charlevoix. Daniel Mathieu, directeur technique à la Fédération de montagne, observe les changements qui se sont produits depuis huit ans sur ce premier grand sentier "moderne", qui va de St-Urbain à la Malbaie: le groupe d'âge s'est elargi vers les deux extrémités, 1'ăge moyen se dêplaçant cntre trente et cinquante ans et les femmes comptent pour 30 a $35 \%$ de ces groupes. Quant aux services," on couche dans des refuges chauffes et certains forfaits prévoient le transport de la nourriture et même des bagages; des chauffeurs déplacent votre auto qui peut ainsi vous attendre à l'autre bout du sentier. La demande est telle que la fédération aimerait bien doubler en plus confortables et en plus grands les refuges existants: on offrirait ainsi un service plus luxueux, des chambres à deux, etc... De tels aménagements répondraient encore mieux aux attentes de la clientèle d'aujourd'hui.

Au total, la grande traversede de Charlevoix aura permis de réaliser 4,200 jours de tourisme. Pour avoir une idée du volume total pour l'ensemble du Québec, il faut se rappeler qu'il existe des sentiers analogues, avec refuges, dans six pares et réserves du
Québec: Mont-Tremblant, Jacques-Cartier, Papineau-Labelle, Mastigouche, du Saguenay, et de la Gaspésie. On peut donc conjecturer que chaque hiver, autour de 20,000 personnes-jours de cette activité sont réalisés, ce qui toucherait un bassin de 5,000 à 7,000 personnes.

\section{De la carte postale à l'écologie}

Un regard du côté de la grande randonnće à pied et des croisières de voile nous ferait voir des réalités semblables. Le plus intéressant est qu'on ne semble pas avoir atteint un plateau: tous constatent que ça bouge, que les courbes sont ascendantes. Il faut se replacer au début des années 70 pour mesurer un peu le chemin parcouru. A cette époque, les habitudes de fréquentation du milieu naturel étaient beaucoup plus stéréotypées et limitées. La chasse et la pêche sont l'usage dominant, qui exclut positivement les autres pratiques des pares et réserves, a l'exception des sites de camping. Le ski alpin se développe tranquillement. La villégiature, l'hôtellerie et le camping sous la tente proposent une forme relativement sédentaire de tourisme. Et on se ballade en auto pour consommer des paysages. Mais c'est au tout début des années 70 que tout se met à bouger, y compris les gens. Dès lors, on ne cessera plus d'entendre parler de pistes, de sentiers de randonnée et de voies cyclables.

La motoneige a poursuivi une évolution parallèle à celle des pratiques non motorisées de plein air. Les motoneigistes representent une clientele importante qui se déplace avec des moyens de transport légers. Après une montée vertigineuse, la popularité de la motoneige se maintient auprès des 740,000 adeptes québécois. Depuis quelques années, plusieurs circuits de randonnée ont été organisés. La région de St-Michel-des-Saints a mềme su en faire un produit touristique important à l'echelle régionale: dès le mois de juin, la clientèle américaine fait le plein des hôtels et motels disponibles. Un développement semblable se fait actuellement en Beauce, région frontalière, selon Raymond Lefebvre de la Fédération de la motoneige. La culture de la motoneige est solidement implantée chez nous avec la gamme complète des activités récréstouristiques: relais, refuges, location, compétitions, rallyes. C'est donc un autre des produits touristiques de deplacement sur le territoire; et le nombre des randonneurs au long cours y serait tout à fait comparable à ceux des autres disciplines.

\section{Un message pour le tourisme québécois}

Cette progression modeste mais toujours soutenue depuis quinze ans du tourisme de longue randonnée est porteuse de plusieurs messages pour le tourisme québécois. En premier, l'émergence de ce secteur confirme la tendance à la segmentation du marche touristique de plein air. Le tourisme fondé sur le contact avec la nature compte
- et pour de bon - plusieurs produits de plus. Il faudra done s' interroger sur l'importance en volume et en possibilités de développement de ces nouveaux produits, qui sont une expression visible de la monté des valeurs écologiques, montée qui est loin d'etre terminé. L'avenir pourra facilement jouer de mauvais tours à notre industrie touristique relice au milieu naturel si nous interprétons mal les contraintes à venir et même les possibilitếs de régression de produits comme la chasse ( $30 \%$ de la population s'y oppose déjà) ou le ski alpin, dont l'augmentation des colits de pratique risque de réduire prochainement le marché.

L'interrogation la plus féconde sur le "message" que ces nouveaux comportements envoient à l'industrie touristique, c'est qu'ils sont l'antidote parfait des aleas du climat québécois. Alors que nos propres médias (météorologues et animateurs radio surtout) sont en guerre ouverte contre nos saisons, le froid de l'hiver, les maringouins et les pluies d'été, voilà que des gens ont répandu des pratiques touristiques qui "battent notre climat sur son propre terrain" " Et d'au moins trois façons. Dejà, ces pratiques ont étendu la saison touristique d'été du début juin à la fin de septembre; la période intensive du canot-camping, par exemple, est le mois de juin. Ensuite, le caractere actif de ce type de tourisme lui font transcender les variations de température d'êté comme d'hiver. Enfin, c'est la varićté méme du milieu naturel avec ses micro-climats et ses milliers de détails à observer qui constituent le menu sensoriel de l'expérience recherchéce. Voilă des gens qui ne s'inquiètent pas de voir passer un nuage!

Le troisième message est un appel à l'intégration de ces produits dans l'industrie touristique régionale et dans le produit touristique québécois. Héritiers d'une situation de divorce où le social et l'économique étaient vus comme des réalités devant nécessairement s'opposer, les promoteurs de ces "nouveaux" produits "on ne peut plus québécois" "se tournent maintenant vers le secteur privé, pour le convaincre de partager les couts avec leur organisation bénévole et le gouvemement qui réduit considérablement son support. Cela se fait depuis longtemps en Europe et aux EtatsUnis. 11 est grand temps que nos gens s'assoient pour se parler, car nous raterons de belles occasions de développement, tant pour le tourisme interne que pour les belles percées qu'il est possible de soutenir en Europe et ailleurs.

Pour finir, une image... Depuis deux ans, des groupes de Suisses, de Français, des Belges, des Allemands, et évidemment de nombreux Américains achètent les cartesguides des rivières canotables. Quand découvrira-t-on chez les chercheurs, qu'il y a "quelque chose la" qui mériterait d'être pese? $f$ 